Article

\title{
Tailorable and Broadband On-Chip Optical Power Splitter
}

\author{
Hyeongpin Kim and Heedeuk Shin * \\ Department of Physics, Pohang University of Science and Technology, Pohang 37673, Korea; \\ khb6566@postech.ac.kr \\ * Correspondence: heedeukshin@postech.ac.kr
}

Received: 20 September 2019; Accepted: 4 October 2019; Published: 10 October 2019

Featured Application: Silicon photonics, Quantum integrated photonic circuits, Photonic integrated circuits.

\begin{abstract}
An on-chip optical power splitter is a key component of photonic signal processing and quantum integrated circuits and requires compactness, wideband, low insertion loss, and variable splitting ratio. However, designing an on-chip splitter with both customizable splitting ratio and wavelength independence is a big challenge. Here, we propose a tailorable and broadband optical power splitter over $100 \mathrm{~nm}$ with low insertion loss less than $0.3 \%$, as well as a compact footprint, based on $1 \times 2$ interleaved tapered waveguides. The proposed scheme can design the output power ratio of transverse electric modes, lithographically, and a selection equation of a power splitting ratio is extracted to obtain the desired power ratio. Our splitter scheme is close to an impeccable on-chip optical power splitter for classical and quantum integrated photonic circuits.
\end{abstract}

Keywords: integrated beam splitter; silicon photonics; tailorable power splitting ratio

\section{Introduction}

Recently, photonic integrated circuits have been intensively investigated for applications in quantum photonics [1], optical interconnection [2], photonic computing [3], and optical communications [4]. Integration of multiple photonic components on a photonic chip requires the development of on-chip photonic integrable components, including waveguides, filters, and optical power splitters. An integrated optical power splitter is an important photonic building block that divides optical power into multiple channels, forms interferometer systems, and taps a small portion of signal [5].

Several approaches have been developed for on-chip power dividers, including Y-junctions [6], directional couplers $[7,8]$, and multimode interferometers $[9,10]$. Conventional Y-junctions typically have a 1-dB insertion loss and a 3-dB splitting ratio due to fabrication imperfections [11,12]. In the case of directional couplers, the power splitting can be designed by controlling the coupling length and distance between the two waveguides, but the coupling ratio strongly depends on the input wavelengths $[8,13]$. Recently, curved directional coupler and its combination with straight directional couplers show small footprints, low loss, and ultra-broadband power splitting, but they need careful design in coupling regions [14,15]. Multimode interferometers not only have several advantages, such as relatively small size and high fabrication tolerances, but also have drawbacks, such as wavelength dependence and insertion loss $[9,16]$. Since the subwavelength grating can engineer refractive index and dispersion properties, a compact, low loss, and ultra-broadband directional couplers are proposed using its design flexibility [17-19], but the gratings are intrinsically sensitive to fabrication errors. Furthermore, a 3-dB beam splitter that uses adiabatically tapered waveguides was demonstrated [20] having a low insertion 
loss $(<0.2 \mathrm{~dB})$, a short coupling length $(5 \mu \mathrm{m})$, and negligible dependency on the input wavelengths, but it has a fixed power split ratio of $50 \%$. Even if these types of on-chip power splitters meet some of the performance requirements, it is a big challenge that an on-chip optical power splitter satisfies all of compactness, low-insertion loss, wavelength independence, and tailorability of the split ratio.

These properties are required not only in classical photonic signal processing but also in quantum photonics. For instance, in quantum photonic integrated circuits, asymmetric interferometers are used to generate time-bin entanglement, but a non-3- $\mathrm{dB}$ power splitter is needed due to propagation loss in longer path [21]. In addition, controlled-NOT gate was demonstrated using directional couplers with 50:50 and 70:30 ratios [3]. However, as the directional couplers used in these experiments have considerable wavelength dependence, precise control of fabrication is required.

In this work, we propose a novel scheme of on-chip photonic power splitters that can meet all the required characteristics of tailorability, wideband, compactness, and low insertion loss. The designed structure is based on $1 \times 2$ interleaved tapered waveguides $(1 \times 2$ ITW $)$ on a silicon-on-insulator platform, and a power split ratio can be determined by moving laterally one of the output tapered waveguides, lithographically. An insertion loss extracted from computational simulation results is less than $0.3 \%$ even if the split ratio changes from $50 \%$ to $1 \%$, and wavelength dependence of the split ratio, as well as the insertion loss, is negligible in the wavelength range from $1.5 \mu \mathrm{m}$ to $1.6 \mu \mathrm{m}$. This scheme is close to impeccable power splitters and can be the most promising photonic power divider for photonic integrated circuit systems and quantum photonics.

\section{Materials and Methods}

The simulations are performed by Lumerical's MODE solution, which offers the 2.5D finite-difference time-domain method. The mesh accuracy used in the simulation is the predefined value 6 in Lumerical, which is small enough for the narrow tips in the tapered region in Figure 1. The time step is about $0.04 \mathrm{fs}$. The boundary conditions are the perfect matched layer condition in the $x$ and $y$-directions and the metal boundary condition in the $z$-direction. This metal boundary condition can reduce the simulation time and does not affect the simulation results as light is tightly confined in the waveguides.
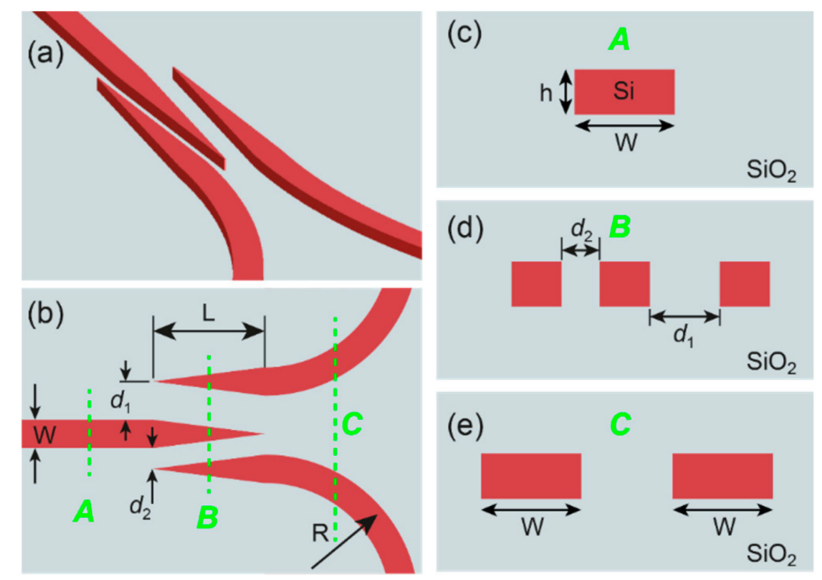

Figure 1. Proposed photonic power splitter based on $1 \times 2$ interleaved tapered waveguides (a) 3D schematic representation of a $1 \times 2$ ITW. (b) Pictorial representation of the splitter from the top-down view. All waveguides have a width of $W=400 \mathrm{~nm}$ and a thickness of $h=220 \mathrm{~nm}$. The tapering length is $\mathrm{L}=15 \mu \mathrm{m}$, and the sharp end of each tapered waveguides has the width of $30 \mathrm{~nm}$. (c)-(e) Cross-sections of the proposed power splitter at several positions. The location of each cross-section is marked on Figure $1 \mathbf{b}$ with green dashed lines as (c) A, (d) B, and (e) C.

A 3D diagram of the proposed beam splitter is illustrated in Figure 1a, and the top-down view of the power splitter system with various geometric parameters is shown in Figure $1 \mathrm{~b}$. The $1 \times 2$ 
interleaved tapered waveguide (ITW) structure consists of an input port with one tapered waveguide and two output ports with tapered waveguides next to the input channel in the opposite direction. The waveguides are tapered in from $400 \mathrm{~nm}$ to $30 \mathrm{~nm}$ across the coupling length $\mathrm{L}(15 \mu \mathrm{m})$, as shown in Figure $1 \mathrm{~b}$. Since the tapering angle of the input and output waveguides is identical, two slot waveguides are created, approaching each other along the propagation direction. The minimum tapering size of $30 \mathrm{~nm}$ is determined from typical and affordable electron beam lithography resolution. Divided optical power comes through two curved waveguides with a bending radius of $30 \mu \mathrm{m}$. The cross-sectional views of the power splitter system at locations A, B, and C in Figure $1 \mathrm{~b}$ are shown in Figure 1c-e, respectively. The silicon waveguides in this scheme are surrounded by a $\mathrm{SiO} 2$ cladding and have a thickness (h) of $220 \mathrm{~nm}$ and a width (W) of $400 \mathrm{~nm}$, as seen in Figure 1c-e.

The performance of this structure is simulated for various geometrical conditions. The gap size between the input tapered waveguide and the upper output waveguide in Figure $1 \mathrm{~b}$ varies lithographically from $d_{1}=150 \mathrm{~nm}$ to $600 \mathrm{~nm}$. By means of changing this gap $d_{1}$, the output ratio can be designed to achieve a desired split ratio. The lower gap of $\mathrm{d}_{2}=150 \mathrm{~nm}$ and the coupling length of $L=15 \mu \mathrm{m}$ are fixed. The gap sizes $d_{1}, d_{2}$ and the coupling length $L$ are larger than those in reference [20]. The gap size of $150 \mathrm{~nm}$ is slightly larger than the decaying depth of the evanescent wave. The gap size of less than $150 \mathrm{~nm}$ can yield complicated coupling between waveguides due to long evanescent tails, and the power splitting becomes sensitive to fabrication errors. The gap size of $150 \mathrm{~nm}$ is large enough to prevent complicated coupling and to achieve tailorable power relatively readily. On the other hand, simulation results show that a beam splitter with larger gap size than $\mathrm{d}_{1}=\mathrm{d}_{2}=150 \mathrm{~nm}$ and shorter coupling length than $\mathrm{L}=15 \mu \mathrm{m}$ significantly increases its insertion loss. With the optimized gap size of $150 \mathrm{~nm}$, the coupling length should be at least $15 \mu \mathrm{m}$ to achieve transition with the fundamental mode and to compensate the loss induced by the large gap.

Note that this method is designed with only one polarization direction in silicon waveguides. The computational simulation is conducted with only transverse electric (TE)-like modes as many photonic integrated circuits support one polarization mode (mostly TE-like mode). In addition, transverse magnetic (TM)-like modes in this scheme have a higher loss, larger wavelength dependence, and a different splitting ratio with TE-like modes.

\section{Results}

Power splitting behaviors in the TE-like mode are presented in Figure 2. The cross-sectional view of the electric field intensity profile of the fundamental TE-like mode at position A of Figure 2a is shown in Figure $2 b$. The cross-sectional views of the fundamental electric field intensity profiles at location $B$ and $C$ for various upper gap sizes $\left(d_{1}=[150 \mathrm{~nm}, 200 \mathrm{~nm}, 315 \mathrm{~nm}]\right)$ are seen in Figure 2c-e, respectively. Note that all the intensity profiles in Figure 2 are normalized with a constant to emphasize the optical power splitting results. The lower gap size is fixed at $150 \mathrm{~nm}$. The top-down views of the power flux density for various $d_{1}$ gap sizes are seen in Figure $2 f-h$, and the extracted output power ratios are (f) 50:50, (g) 70:30, and (h) 90:10 at $d_{1}=[150 \mathrm{~nm}, 200 \mathrm{~nm}, 315 \mathrm{~nm}]$, respectively. 
(a)
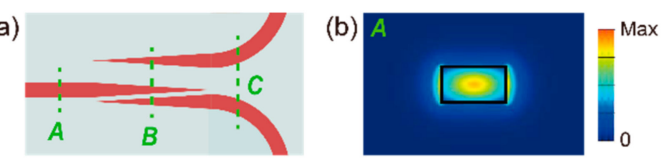

(c)
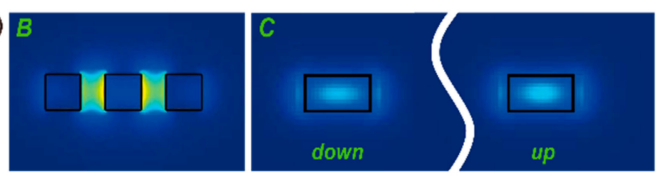

(d)
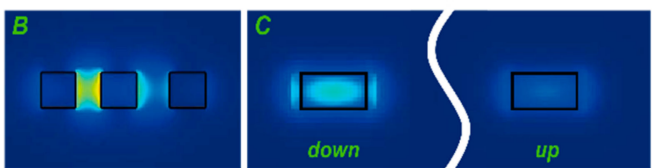

(e) $B$
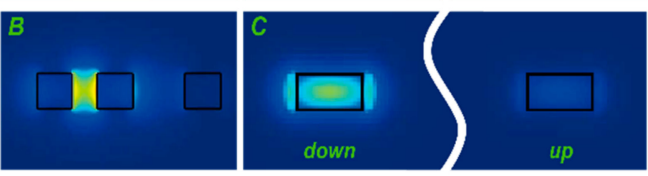

(f)

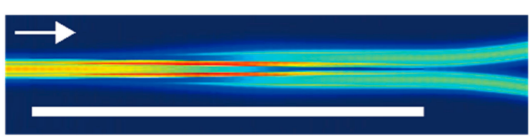

(g)

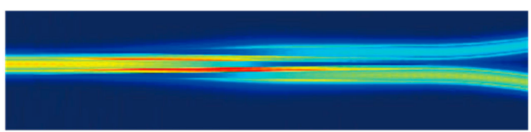

(h)

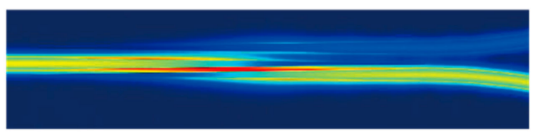

Figure 2. Simulation results showing the split mechanism of the proposed optical power splitter. (a) Pictorial top-down view of the splitter. Here, the upper gap size, $\mathrm{d}_{1}$, varies to control the output power ratio. The lower gap size, $\mathrm{d}_{2}$, is fixed at $150 \mathrm{~nm}$. (b) Electric field intensity profile for fundamental transverse electric (TE)-like mode at the cross-section A on Figure 2a. (c)-(e) Electric field intensity profiles of the splitter for various gap sizes of $d_{1}$ as (c) $150 \mathrm{~nm}$, (d) $200 \mathrm{~nm}$, or (e) $315 \mathrm{~nm}$. The fundamental modes at cross sections $B$, and the output electric field intensities at cross sections $C$ are presented. (f)-(h) Top-down view of the power flux density for various gap sizes. The power ratios are (f) 50:50, (g) 70:30, and (h) 90:10. The white arrow and bar in (f) indicates the energy flow and $15 \mu \mathrm{m}$.

From the boundary conditions of Maxwell's equations, the discontinuity of the electric fields at the boundaries of two adjacent waveguides amplifies the electric field in the gap, referred to as a slot waveguide mode [22]. Therefore, input light arriving at the coupling region slowly converges to double slot waveguide mode, as seen in the left-hand figures of Figure 2c-e. These show the fundamental modes of the electric field intensity profile at the cross section B in Figure 2a. At the end of the coupling section the electric field is strongly confined to the two output waveguides, splitting the optical power as a function of the gap size, $\mathrm{d}_{1}$. The right-hand figures of Figure $2 \mathrm{c}-\mathrm{e}$ show the intensity profile of the two output ports at section $C$ of Figure $2 a$, proving that the optical power through the upper (lower) output port decreases (increases) as enlarging the upper gap size, $\mathrm{d}_{1}$.

In the coupling region, in order to avoid the excitation to higher modes, it is required to maintain a large difference between the effective index of fundamental and secondary modes. The width of input/output waveguides is optimized to $400 \mathrm{~nm}$ in order to keep the index difference with low propagation and bending loss [20]. However, in our scheme, the asymmetry structure by $d_{1} \neq d_{2}$ and the large gap size reduce the effective index difference between the fundamental mode and the higher modes, but the excitation to unwanted higher modes can be prevented by designing the coupling length [23]. To maintain the fundamental mode transition, the coupling length of $15 \mu \mathrm{m}$ is determined from the careful simulations. 
A beam splitter with a specific power ratio can be designed by controlling the upper gap size. In Figure 3a, the simulation results of the transmittance through the upper, lower, and the total output channels are shown for the upper gap size. As the upper gap size increases, the coupling with the upper (lower) output waveguide exponentially decreases (increases) at the same rate, respectively. The coupling ratio is defined as $\mathrm{R}=\mathrm{P}_{\mathrm{up}} /\left(\mathrm{P}_{\mathrm{up}}+\mathrm{P}_{\text {down }}\right)$. Based on the simulation results in Figure $3 \mathrm{a}$, an upper gap size $d_{1}$ for a desired coupling ratio $R$ with a fixed lower gap size $d_{2}=150 \mathrm{~nm}$ is given by

$$
d_{1}=\frac{1}{0.01} \times \ln \left(\frac{2.25}{R-0.004}\right)
$$

where the coupling ratio $R$ has a value between 0.004 and 0.5 and this equation is quoted for $d_{1}$ in nanometers. This equation can be used to determine an upper gap size for a desired coupling ratio. For example, to design a 90:10 beam splitter, the coupling ratio should be $\mathrm{R}=0.1$, and the required upper gap size is $d_{1}=315 \mathrm{~nm}$ with a fixed lower gap size of $d_{2}=150 \mathrm{~nm}$. Figure $3 b$ shows the simulated insertion loss of the structure, $\left[\left[\mathrm{P}_{\text {in }}-\mathrm{P}_{\text {up }}-\mathrm{P}_{\text {down }}\right] / \mathrm{P}_{\text {in }}\right]$. By increasing the upper gap size from $150 \mathrm{~nm}$ to $600 \mathrm{~nm}$, the insertion loss increases from $0.1 \%$ to $0.25 \%(\sim 0.01 \mathrm{~dB})$, which is negligibly low considering fabrication imperfections. In practice, light scattering from side-wall surfaces or fabrication error will be the dominant loss mechanisms.

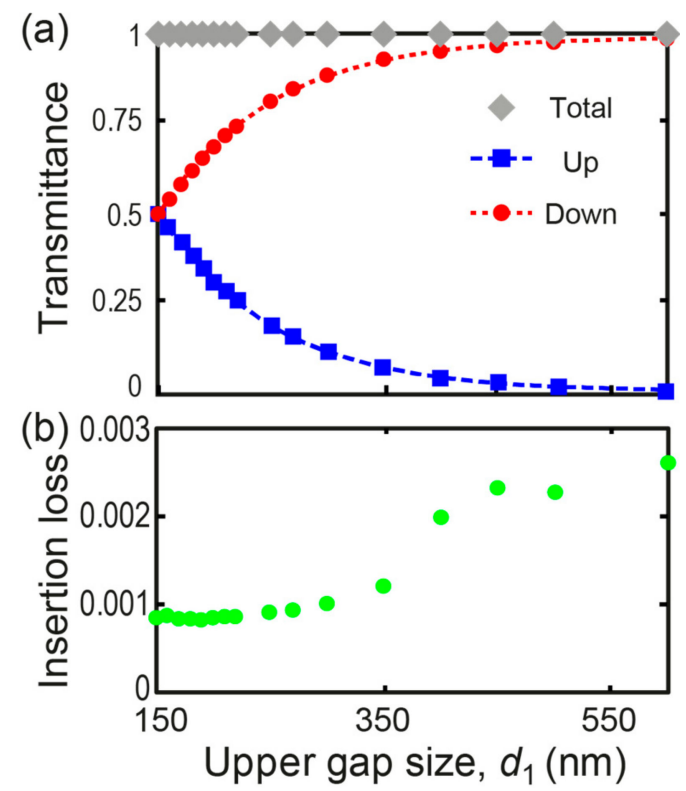

Figure 3. Transmittance and insertion loss against the upper gap size, $d_{1}$. (a) The gray diamonds represent the total output power, and the red circles (blue squares) indicate the downward (upward) output, respectively. The theoretical fit obtained using an exponential function is shown as dotted lines atop the simulation data (red circles and blue squares). (b) Insertion loss versus upper gap size. While the upper gap size varies from $150 \mathrm{~nm}$ to $600 \mathrm{~nm}$, the insertion loss of each gap is less than $0.3 \%$.

Figure 4 shows the transmittance through the upper and lower channels against input wavelength. The computational simulation is performed over a wavelength range of $1.5 \mu \mathrm{m}$ to $1.6 \mu \mathrm{m}$ for various upper gap sizes $\left(\mathrm{d}_{1}=[150 \mathrm{~nm}, 200 \mathrm{~nm}, 315 \mathrm{~nm}, 590 \mathrm{~nm}]\right)$ corresponding to the power splitting ratios of 50:50, 70:30, 90:10, and 99:1, respectively. The simulated power split ratio has a deviation of less than $6.5 \%$ from its desired value within the overall simulation wavelength range. The profile of evanescent waves depends on the input wavelength, which may cause wavelength dependence. To reduce wavelength dependence, the lower gap size is optimized to $150 \mathrm{~nm}$, which is larger than the characteristic decaying length of the input light but small enough to support slot waveguide modes 
with weak wavelength dependence. The larger gap size reduces wavelength dependence, but leads to high optical power leakage.

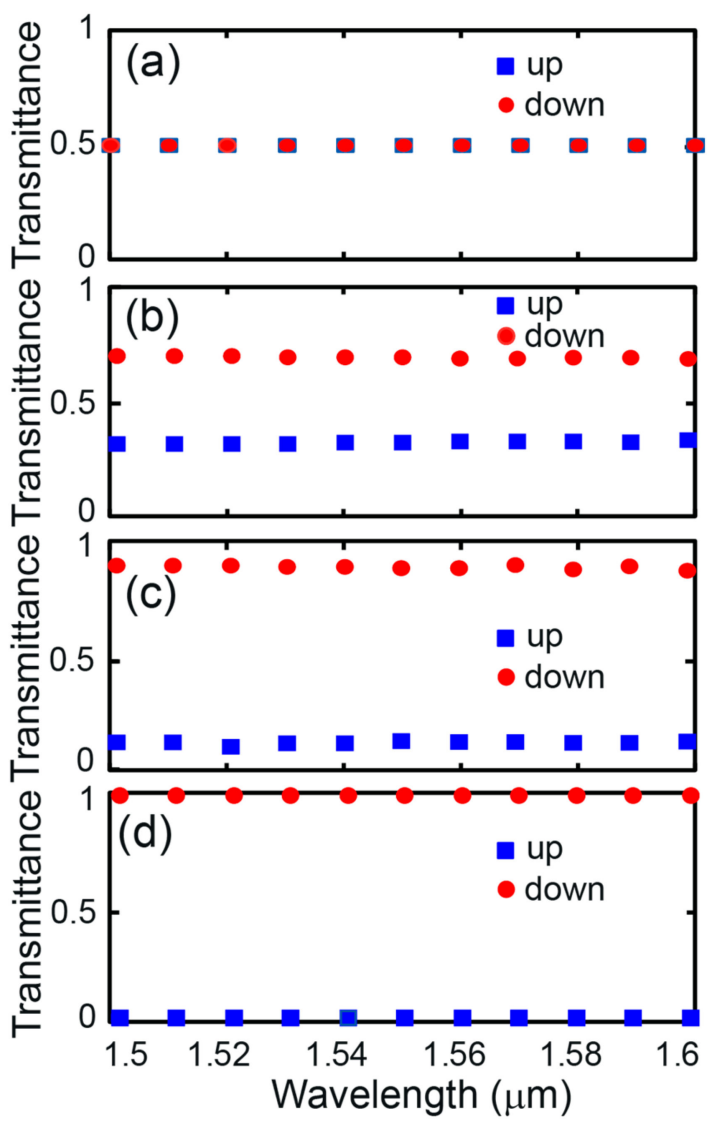

Figure 4. Transmittance against input wavelength at several upper gap sizes. (a) $150 \mathrm{~nm}$ (50:50 power splitter), (b) $200 \mathrm{~nm}$ (70:30 power splitter), (c) $315 \mathrm{~nm}$ (90:10 power splitter), and (d) $590 \mathrm{~nm}$ (99:1 power splitter) of the upper gap sizes were simulated. The red circles (blue squares) represent the output power ratios through the lower (upper) channel, respectively.

The coupling ratio deviation is defined by $\left(\frac{\operatorname{abs}\left(\mathrm{P}_{\text {down, target }}-\mathrm{P}_{\text {down }}\right)}{\mathrm{P}_{\text {down, target }}} \times 100\right) \%$, and the maximum deviations in wavelength range between $1.5 \mu \mathrm{m}$ and $1.6 \mu \mathrm{m}(1.2 \mu \mathrm{m}-1.8 \mu \mathrm{m})$ are $0.49 \%(2.54 \%)$ for $50: 50,2.85 \%(12.30 \%)$ for $30: 70,0.27 \%(7.11 \%)$ for $10: 90$, and $0.86 \%(1.20 \%)$ for $1: 99$. Due to the slowly varying material refractive index in wavelength, the evanescent profile, as well as the splitting ratio, change slowly in wavelength. However, even in $1.2 \mu \mathrm{m}-1.8 \mu \mathrm{m}$ range, the maximum deviation is about $10 \%$, showing ultra-broadband tailorability compared to recent methods of broadband power splitters $[14,18]$.

\section{Discussion}

Note that the geometry of the proposed system has typical size values and materials that are used in common photonic integrated circuit technologies. The input and output waveguides can be readily fabricated using excimer laser photolithography, but the feature size of $30 \mathrm{~nm}$ at the tapering tips is too small for typical photolithography. Electron beam lithography is one way to obtain such a resolution, but it is not a cost effective way. Alternative method is hybrid lithography where photolithography is used for the entire waveguide system and electron beam lithography is used only for the high-resolution region [24]. 
The waveguide width can be slightly reduced or enlarged by fabrication error, which can result in a different beam-splitter-gap size from its original design. In our scheme, the output split ratio is determined by the relative gap size between the upward and the downward waveguide. If all waveguides shrink or expand in the same way, the difference between the two gap sizes would not change, maintaining the split ratio. To test the effect of this transformation on the splitting ratio, we repeat the computational simulations with an acceptable waveguide width tolerance of $\pm 10 \mathrm{~nm}$ in electron beam lithography. From the simulation results, the transmittance of the output changes by up to $1 \%$ from the desired splitting ratio values, which can be negligible. This result shows that our optical power splitter scheme is robust to this type of fabrication errors. In addition, when the width or height of the input/output waveguides needs to change under certain experimental conditions, the size of the gap and the selection equation of a power splitting ratio should slightly differ from the current state. Designing a precise beam splitter, though not strictly necessary, should be considered with new conditions.

In this simulation, only the TE mode is considered. In the TM mode, the evanescent electric field exists at the top and bottom of the waveguides, and the mode profile in the coupling region is complicated and very sensitive to wavelength and geometry dimension variance. In relevant simulations, we observe that the relationship between the gap size and power splitting ratio in the TM mode is different from Equation (1) and is not as simple as that in the TE mode case. We could not find a meaningful and straightforward expression of the relationship as the ratio is too sensitive to the gap size. In addition, we consider the linear optical properties of the proposed design by excluding the nonlinear absorption, power-dependent refractive index, and thermal heating. Further research about the nonlinear optical behaviors of this device is needed.

Recently, other materials, such as silicon nitride, aluminum nitride, and lithium niobate, are intensively investigated in the fields of photonic integrated circuits. Our suggested structure can be employed not only in silicon but also in other platforms. Due to the different refractive index, mode size, and evanescent profile on other platforms, the parameters in Equation (1) differ from those in silicon than silicon, and further research is required.

\section{Conclusions}

A $1 \times 2$ tailorable and broadband beam splitter with low insertion loss is proposed for the silicon-on-insulator platform. The designed structure is based on $1 \times 2$ interleaved tapered waveguides with a coupling length of $15 \mu \mathrm{m}$, and the power split ratio can be determined by the asymmetric gap size between the upper and lower output channels. The relation between the split ratio, $R$, and the upper gap size, $d_{1}$, is extracted from the simulation results with a fixed lower gap size of $150 \mathrm{~nm}$. With this equation, a tailorable beam splitter of a desired coupling ratio can be designed. The proposed beam splitter scheme has most of the performance requirements of on-chip optical beam splitters and can be the most promising on-chip optical power divider in various photonic applications of classical and quantum photonic integrated circuits.

\section{Patents}

A patent according to this result has been registered at the Korean Intellectual Property Office (Reg. \#: 10-1844987, Registration date: 03/28/2018).

Author Contributions: Simulation, H.K. and H.S.; writing-original draft preparation, H.K.; writing-review and editing, H.S.; supervision, H.S.

Funding: This work was supported by the National Research Foundation of Korea (Grant No. NRF-2017R1C1B2011113).

Acknowledgments: This work is partially supported by the Affiliated Institute of ETRI.

Conflicts of Interest: The authors declare no conflict of interest. 


\section{References}

1. Reimer, C.; Kues, M.; Roztocki, P.; Wetzel, B.; Grazioso, F.; Little, B.E.; Chu, S.T.; Johnston, T.; Bromberg, Y.; Caspani, L.; et al. Generation of multiphoton entangled quantum states by means of integrated frequency combs. Science 2016, 351, 1176-1180. [CrossRef] [PubMed]

2. Ohashi, K.; Nishi, K.; Shimizu, T.; Nakada, M.; Fujikata, J.; Ushida, J.; Toru, S.; Nose, K.; Mizuno, M.; Yukawa, H.; et al. On-chip optical interconnect. Proc. IEEE 2009, 97, 1186-1196. [CrossRef]

3. Poot, M.; Schuck, C.; Ma, X.; Guo, X.; Tang, H.X. Design and characterization of integrated components for SiN photonic quantum circuits. Opt. Express 2016, 24, 6843. [CrossRef] [PubMed]

4. Luo, Y.; Yu, Y.; Ye, M.; Sun, C.; Zhang, X. Integrated dual-mode $3 \mathrm{~dB}$ power coupler based on tapered directional coupler. Sci. Rep. 2016, 6, 23516. [CrossRef] [PubMed]

5. Subbaraman, H.; Xu, X.; Hosseini, A.; Zhang, X.; Zhang, Y.; Kwong, D.; Chen, R.T. Recent advances in silicon-based passive and active optical interconnects. Opt. Express 2015, 23, 2487. [CrossRef] [PubMed]

6. Sakai, A.; Fukazawa, T.; Baba, T. Low loss ultra-small branches in a silicon photonic wire waveguide. IEICE Trans. Electron. 2002, E85-C, 1033-1038.

7. Takagi, A.; Jinguji, K.; Kawachi, M. Wavelength Characteristics of $(2 \times 2)$ Optical Channel-Type Directional Couplers with Symmetric or Nonsymmetric Coupling Structures. J. Light. Technol. 1992, 10, 735-746. [CrossRef]

8. Yamada, H.; Chu, T.; Ishida, S.; Arakawa, Y. Optical directional coupler based on Si-wire waveguides. IEEE Photonics Technol. Lett. 2005, 17, 585-587. [CrossRef]

9. Thomson, D.J.; Hu, Y.; Reed, G.T.; Fedeli, J.M. Low loss MMI couplers for high performance MZI modulators. IEEE Photonics Technol. Lett. 2010, 22, 1485-1487. [CrossRef]

10. Blahut, M.; Opilski, A. Multimode interference structures-A new way of passive elements technology for photonics. Opto-Electron. Rev. 2001, 9, 293-300.

11. Zhang, Y.; Yang, S.; Lim, A.E.J.; Lo, G.Q.; Galland, C.; Baehr-Jones, T.; Hochberg, M. A compact and low loss Y-junction for submicron silicon waveguide. Opt. Express 2013, 21, 1310-1316. [CrossRef]

12. Raghuwanshi, S.K.; Kumar, S.; Kumar, V.; Chack, D. Propagation Study of Y-branch Having Inbuilt Optical Splitters and Combiner Using Beam Propagation Method. In Proceedings of the The 32nd PhotonIcs and Electromagnetics Research Symposium (PIERS), Moscow, Russia, 19-23 August 2012; pp. 720-724.

13. Lu, Z.; Wang, Y.; Zhang, F.; Jaeger, N.A.F.; Chrostowski, L. Wideband silicon photonic polarization beamsplitter based on point-symmetric cascaded broadband couplers. Opt. Express 2015, 23, 29413. [CrossRef] [PubMed]

14. Chen, G.F.R.; Ong, J.R.; Ang, T.Y.L.; Lim, S.T.; Png, C.E.; Tan, D.T.H. Broadband Silicon-On-Insulator directional couplers using a combination of straight and curved waveguide sections. Sci. Rep. 2017, 7, 4-11. [CrossRef] [PubMed]

15. Chen, S.; Shi, Y.; He, S.; Dai, D. Low-loss and broadband $2 \times 2$ silicon thermo-optic Mach-Zehnder switch with bent directional couplers. Opt. Lett. 2016, 41, 836-839. [CrossRef] [PubMed]

16. Solehmainen, K.; Kapulainen, M.; Harjanne, M.; Aalto, T. Adiabatic and multimode interference couplers on Silicon-on-Insulator. IEEE Photonics Technol. Lett. 2006, 18, 2287-2289. [CrossRef]

17. Yun, H.; Wang, Y.; Zhang, F.; Lu, Z.; Lin, S.; Chrostowski, L.; Jaeger, N.A.F. Broadband $2 \times 2$ adiabatic 3 dB coupler using silicon-on-insulator sub-wavelength grating waveguides. Opt. Lett. 2016, 41, 3041. [CrossRef] [PubMed]

18. Yun, H.; Chrostowski, L.; Jaeger, N.A.F. Ultra-broadband $2 \times 2$ adiabatic 3 dB coupler using subwavelength-grating-assisted silicon-on-insulator strip waveguides. Opt. Lett. 2018, 43, 1935. [CrossRef] [PubMed]

19. Xu, L.; Wang, Y.; Kumar, A.; El-Fiky, E.; Mao, D.; Tamazin, H.; Jacques, M.; Xing, Z.; Saber, M.G.; Plant, D.V. Compact high-performance adiabatic 3-dB coupler enabled by subwavelength grating slot in the silicon-on-insulator platform. Opt. Express 2018, 26, 29873. [CrossRef]

20. Wang, Y.; Gao, S.; Wang, K.; Skafidas, E. Ultra-broadband and low-loss 3 dB optical power splitter based on adiabatic tapered silicon waveguides. Opt. Lett. 2016, 41, 2053-2056. [CrossRef]

21. Xiong, C.; Zhang, X.; Mahendra, A.; He, J.; Choi, D.Y.; Chae, C.J.; Marpaung, D.; Leinse, A.; Heideman, R.G.; Hoekman, M.; et al. Compact and reconfigurable silicon nitride time-bin entanglement circuit. Optica 2015, 2, 724. [CrossRef] 
22. Almeida, V.R.; Xu, Q.; Barrios, C.A.; Lipson, M. Guiding and confining light in void nanostructure. Opt. Lett. 2004, 29, 1209. [CrossRef] [PubMed]

23. Dai, D.; Tang, Y.; Bowers, J.E. Mode conversion in tapered submicron silicon ridge optical waveguides. Opt. Express 2012, 20, 13425. [CrossRef] [PubMed]

24. Pease, R.F.; Chou, S.Y. Lithography and other patterning techniques for future electronics. Proc. IEEE 2008, 96, 248-270. [CrossRef]

(C) 2019 by the authors. Licensee MDPI, Basel, Switzerland. This article is an open access article distributed under the terms and conditions of the Creative Commons Attribution (CC BY) license (http://creativecommons.org/licenses/by/4.0/). 